\title{
USO DE DIFERENTES PLASTIFICANTES NA PRODUÇÃO DE BIOFILMES À BASE DE AMIDO DE PINHÃO
}

\author{
R. M. DAUDT ${ }^{1}$, V. F. TASENDE ${ }^{2}$, I. CAFRUNI ${ }^{2}$, R. C. S. THYS ${ }^{2}$, F. CLADERA-OLIVERA ${ }^{2}$, I. C. \\ KÜLKAMP-GUERREIRO ${ }^{3}$ e L. D. F. MARCZAK ${ }^{1}$ \\ ${ }^{1}$ Universidade Federal do Rio Grande do Sul, Departamento de Engenharia Química \\ ${ }^{2}$ Universidade Federal do Rio Grande do Sul , Instituto de Ciência e Tecnologia de Alimentos \\ ${ }^{3}$ Universidade Federal do Rio Grande do Sul, Faculdade de Farmácia \\ E-mail para contato: redaudt@gmail.com
}

\begin{abstract}
RESUMO - A indústria de alimentos atualmente busca melhorar a qualidade dos alimentos reduzindo os resíduos de embalagem e incentivando o uso de novos materiais. Os biofilmes são filmes finos preparados a partir de materiais biológicos renováveis com a função de agir como barreira a elementos externos, proteger o produto e aumentar a sua vida de prateleira. $\mathrm{O}$ objetivo deste trabalho foi elaborar filmes biodegradáveis à base de amido de pinhão e testar possíveis plastificantes. Foram utilizados como plastificantes sorbitol, glicerol, sacarose e xarope de glicose nas proporções de 4 e 5 g/100g. O amido de pinhão variou de 2 a $6 \mathrm{~g} / 100 \mathrm{~g}$ e água foi adicionada até completar $100 \mathrm{~g}$. A solução filmogênica foi aquecida à temperatura de $70^{\circ} \mathrm{C}$, em banho-maria por $15 \mathrm{~min}$. As soluções foram espalhadas em placas de Petri, secas em estufa a $40^{\circ} \mathrm{C}$ por $24 \mathrm{~h}$ e, posteriormente, colocadas em ambiente com alta umidade relativa (80\%) para facilitar a remoção do biofilme da placa. Estes filmes foram avaliados conforme aparência visual e caracterizados quanto à cor, opacidade e espessura, sendo o sorbitol considerado como o melhor plastificante.
\end{abstract}

\section{INTRODUÇÃO}

Hoje em dia há um interesse cada vez maior em aproveitar a biodiversidade regional, com ênfase no seu uso sustentável. O fortalecimento industrial dos chamados fitoprodutos pode criar condições que permitam, a médio e longo prazo, investir na exploração racional da biodiversidade brasileira para o desenvolvimento de produtos de maior valor agregado, contribuindo para a identificação de oportunidades no âmbito do agronegócio.

O pinhão é a semente da Araucaria angustifolia, uma espécie de conífera nativa do Brasil muito importante economicamente, além de ser uma boa fonte de amido (aproximadamente $36 \%$ em base úmida) (Cordenunsi et al., 2004). Ao desenvolver técnicas de conservação e industrialização, a comercialização e o uso do pinhão em outras épocas do ano, além da estação de produção, pode tornar o mercado mais atraente e incentivar a sua extração e comercialização por parte dos produtores rurais (dos Santos et al., 2002). Assim, a comercialização do pinhão poderia representar uma fonte alternativa de renda para os produtores rurais além de promover o cultivo do pinheiro do Paraná e 
desestimular seu corte ilegal, contribuindo para a preservação da espécie.

Uma alternativa de aplicação do pinhão pode ser a utilização do amido presente em grande quantidade nesta semente e já estudado anteriormente (Bello-Pérez et al., 2006; Thys et al., 2008; Thys et al., 2010; Spada et al., 2012; Daudt et al., 2014) como base para a produção de biofilmes comestíveis. Os filmes utilizados atualmente são embalagens plásticas provenientes de derivados de petróleo, que podem acarretar vários problemas ambientais por não serem biodegradáveis em curto prazo (Sorrentino et al., 2007).

Para a confecção de biofilmes à base de amido é necessária a utilização de plastificantes, com o objetivo de deixar o filme maleável e passível de aplicação. Bons plastificantes utilizados para esse fim pode ser glicerol, sorbitol, sacarose e açúcar invertido (Mali et al., 2006; Kechichian et al., 2010; Kammoun et al., 2013; Maniglia et al., 2014).

Considerando o exposto anteriormente, o desenvolvimento de filmes comestíveis à base de amido de pinhão atende à necessidade da indústria alimentar de gerar embalagens biodegradáveis e ainda empregar um produto de maneira sustentável. Assim, o objetivo deste trabalho foi testar a adição de diferentes plastificantes às soluções de amido de pinhão a fim de se obter biofilmes maleáveis que pudessem ser aplicados posteriormente como embalagem em alimentos.

\section{MATERIAIS E MÉTODOS}

\subsection{Matéria-prima}

Os pinhões (sementes da Araucaria angustifolia) foram obtidos em mercado local (Porto Alegre, RS), selecionados, lavados em água corrente, secos a temperatura ambiente por aproximadamente $24 \mathrm{~h}$, colocados em sacos de polietileno de $2 \mathrm{~kg}$ e armazenados em freezer doméstico $\left(\sim-15^{\circ} \mathrm{C}\right)$.

Os plastificantes utilizados foram: sorbitol (uso industrial, 70\%, Próton Química), glicerol (glicerina P.A., Nuclear), sacarose (sacarose P.A., ACS Synth) e xarope de glicose (xarope de glicose de milho GLUCOSUL).

\subsection{Extração do amido de pinhão nativo}

A extração do amido de pinhão nativo foi realizada utilizando água como solvente, de acordo com a metodologia proposta por Bello-Pérez et al. (2006) e modificada por Daudt et al. (2014).

\subsection{Desenvolvimento dos biofilmes}

Os biofilmes foram desenvolvidos através do método de casting de acordo com Fakhoury et al. (2012). A Tabela 1 apresenta as proporções de amido e plastificante utilizados nas formulações propostas. $\mathrm{O}$ amido e o plastificante foram pesados e água destilada foi acrescentada até completar a 
massa final de $100 \mathrm{~g}$. Foram utilizados sorbitol, glicerol, sacarose e xarope de glicose como plastificantes, sendo que o xarope de glicose foi utilizado apenas na proporção de $4 \mathrm{~g} / 100 \mathrm{~g}$.

Tabela 1 - Proporções de amido e plastificante utilizados no desenvolvimento dos biofilmes

\begin{tabular}{|c|c|c|c|c|c|c|c|c|c|c|}
\hline Plastificante (g/100g) & \multicolumn{3}{|c|}{4,0} & \multicolumn{5}{c|}{5,0} \\
\hline Amido de pinhão (g/100g) & 2,0 & 3,0 & 4,0 & 5,0 & 6,0 & 2,0 & 3,0 & 4,0 & 5,0 & 6,0 \\
\hline
\end{tabular}

A solução foi aquecida em banho termostático (NT 232, Novatecnica, Brasil) a $70^{\circ} \mathrm{C}$, por 15 min. Em seguida, a solução filmogênica formada foi vertida em placas de Petri limpas e secas $\left(0,5 \mathrm{~g} / \mathrm{cm}^{2}\right)$ e colocadas em estufa $\left(\mathrm{B} 3 \mathrm{CBE}\right.$, De Leo, Brasil) a $40^{\circ} \mathrm{C}$ por $24 \mathrm{~h}$ para secar. Algumas das formulações necessitaram de acondicionamento em câmara de controle de fermentação (AC20T, VENANCIO, Brasil) com UR de $80 \%$ para facilitar a remoção das placas. Após a remoção, os filmes foram acondicionados individualmente em refrigerador $\left(5^{\circ} \mathrm{C}\right)$ até realização das análises.

\subsection{Caracterização dos biofilmes} espessura.

Os biofilmes obtidos foram avaliados visualmente e de acordo com a cor, opacidade e

A cor dos biofilmes foi medida em colorímetro (Konica Minolta ${ }^{\circledR}$, modelo Chroma Meter CR400, Singapura) em três diferentes pontos. Os parâmetros colorimétricos $L^{*}, a^{*}$ e $b^{*}$ da escala CIELAB, determinados pela Comission International de l'Eclairarge (CIE) foram obtidos através do espectro de refletância da amostra. (Minolta, 1998).

A opacidade foi medida diretamente com espectrofotômetro portátil (Spectro-guide sphere gloss, BYK, USA) em três pontos diferentes dos biofilmes e expressos em percentual.

A espessura foi medida em 9 pontos diferentes dos biofilmes com micrômetro digital (IP65, Mitutoyo, Brasil).

Os resultados foram analisados utilizando Análise de Variância (ANOVA) e teste de Tukey $(\mathrm{p}<0,05)$ para verificar diferenças estatisticamente significativas.

\section{RESULTADOS E DISCUSSÃO}

A partir das variações do tipo de plastificantes, bem como das suas quantidades e quantidade de amido de pinhão foram obtidos 35 (trinta e cinco) diferentes biofilmes. A Figura 1 ilustra alguns dos biofilmes obtidos com diferentes plastificantes, cada um representando um grupo com um tipo de plastificante. Pode-se perceber através da análise visual que os filmes confeccionados com sorbitol (A) e glicerol (B) apresentaram melhor aparência, maior uniformidade e maleabilidade. Diversos autores reportaram o uso de sorbitol (Sobral et al., 2001; Gialamas et al., 2010; Al-Hassan e Norziah, 
2012; Maniglia et al., 2014) e glicerol (Garcia et al., 2006; Mali et al., 2006; Salmieri e Lacroix, 2006; Chillo et al., 2008; Vásconez et al., 2009; Souza et al., 2012; Kammoun et al., 2013) em seus estudos como agentes plastificantes em biofilmes.

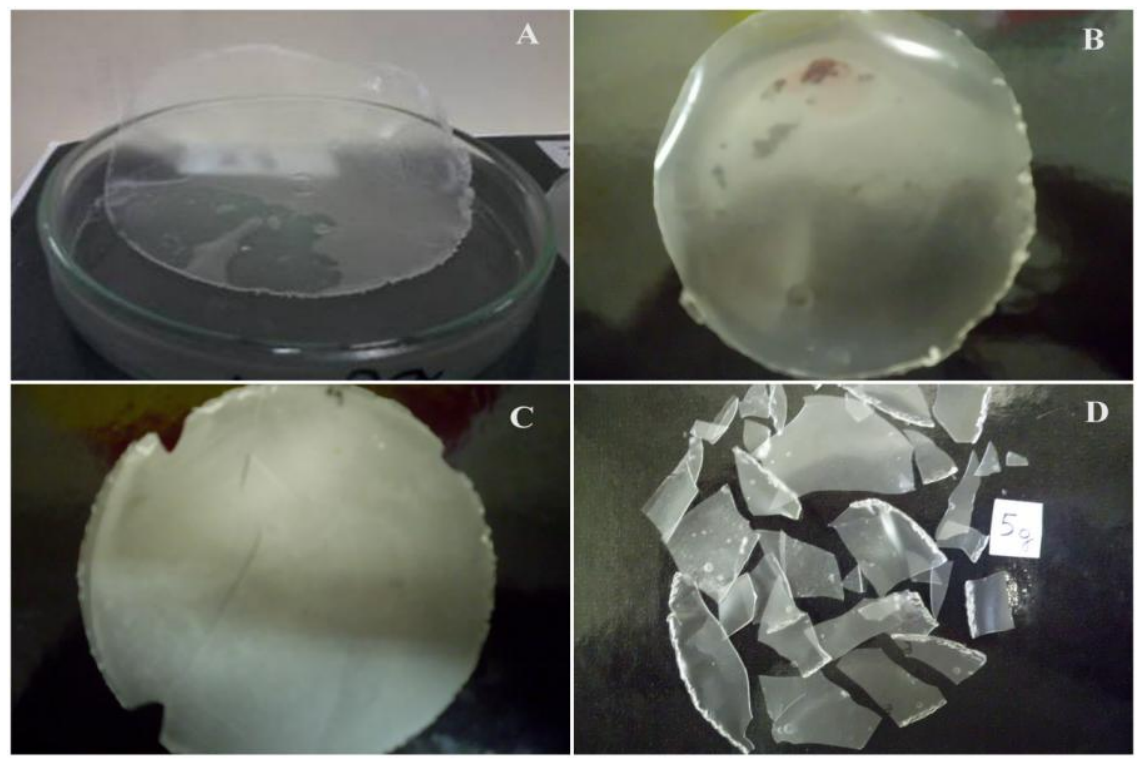

Figura 1 - Fotos dos biofilmes com $4 \mathrm{~g}$ sorbitol/100 g e $4 \mathrm{~g}$ amido de pinhão/100 g (A), $4 \mathrm{~g}$ glicerol/100 g e $4 \mathrm{~g}$ amido de pinhão/100 g (B), $4 \mathrm{~g}$ sacarose/100 g e $4 \mathrm{~g}$ amido de pinhão/100 $\mathrm{g}(\mathrm{C})$, $4 \mathrm{~g}$ xarope de glicose/100 g e $5 \mathrm{~g}$ amido de pinhão/100 g (D).

As melhores formulações utilizando glicerol foram às que continham $4 \mathrm{~g}$ de glicerol/100 $\mathrm{g}$ de solução filmogênica e maiores quantidades de amido de pinhão. As formulações com menores quantidades de amido formaram filmes com textura pegajosa e estrutura frágil. Os filmes de sorbitol mais uniformes e que apresentaram menor variação da espessura foram àqueles compostos por 2 a $4 \mathrm{~g}$ de amido de pinhão/100 g de solução filmogênica.

A utilização isolada da sacarose e do xarope de glicose como plastificantes não permitiram a formação de filmes maleáveis. Os filmes com sacarose ficaram esbranquiçados, opacos e pouco maleáveis e os filmes com xarope de glicose ficaram quebradiços, demonstrando que estes dois ingredientes, quando utilizados nas condições descritas pelo presente trabalho não têm função plastificante. Kechician et al. (2010) utilizaram sacarose e açúcar invertido combinados como plastificante na proporção 1:2 (sacarose:açúcar invertido) em filmes à base de fécula de mandioca e obtiveram um filme base bom para a incorporação de ingredientes antimicrobianos naturais. $\mathrm{O}$ xarope de glicose possui características semelhantes ao açúcar invertido e foi utilizado neste trabalho por ser uma alternativa com menor custo. $\mathrm{O}$ uso combinado destes dois ingredientes poderia ser uma boa alternativa, uma vez que o xarope de glicose poderia evitar a cristalização da sacarose e, juntamente com a sacarose, contribuir para a formação de um filme mais transparente e maleável. Porém, separadamente ambos os ingredientes não apresentaram boas características como plastificantes. 


\section{9 a 22 de outubro de 2014 \\ Florianópolis/SC}

A Tabela 2 apresenta os resultados das análises de cor, opacidade, espessura e análise subjetiva dos biofilmes produzidos, observada através da aparência visual dos mesmos. Os parâmetros de cor, no geral, indicaram filmes claros, com altos valores de luminosidade ( $\mathrm{L}^{*}$ ) e valores das coordenadas cromáticas ( $\mathrm{a}^{*} \mathrm{e} \mathrm{b}^{*}$ ) próximos ao zero. A opacidade, por sua vez, demonstrou que os biofilmes preparados com sacarose apresentaram valores mais altos, ou seja, menos transparentes, confirmando o que já havia sido constatado visualmente. Os filmes confeccionados com sorbitol e glicerol apresentaram maior transparência com valores mais baixos para opacidade, em torno de $5 \%$ e, devido a não uniformidade e por estarem quebradiços, essa medida não foi possível de realizar nos filmes confeccionados com xarope de glicose. A espessura aumentou de acordo com o aumento da quantidade de sólidos totais presentes nas formulações.

Tabela 2 - Resultados das análises de cor, opacidade, espessura e aparência visual dos biofilmes produzidos com diferentes plastificantes

\begin{tabular}{|c|c|c|c|c|c|c|c|}
\hline \multicolumn{2}{|c|}{ Biofilme $^{\#}$} & $L^{*}$ & $a^{*}$ & $b^{*}$ & Opacidade (\%) & $\begin{array}{c}\text { Espessura } \\
(\mu \mathrm{m})\end{array}$ & $\begin{array}{l}\text { Aparên } \\
\text {-cia }\end{array}$ \\
\hline \multirow{5}{*}{$4 S_{\circ}$} & $2 \mathrm{~A}$ & $92,09 \pm 0,79^{\mathrm{a}, \mathrm{b}, \mathrm{c}}$ & $0,45 \pm 0,03^{\mathrm{g}, \mathrm{h}, \mathrm{i}, \mathrm{j}, \mathrm{k}}$ & $0,73 \pm 0,10^{\mathrm{k}, \mathrm{l}}$ & $5,61 \pm 0,26^{h, i}$ & $168 \pm 10^{\circ}$ & ++ \\
\hline & $3 \mathrm{~A}$ & $91,72 \pm 0,74^{\mathrm{a}, \mathrm{b}, \mathrm{c}, \mathrm{d}, \mathrm{e}, \mathrm{f}}$ & $0,71 \pm 0,06^{\mathrm{c}, \mathrm{d}}$ & $0,77 \pm 0,15^{\mathrm{k}}$ & $5,66 \pm 0,08^{\text {g.h,i }}$ & $210 \pm 18^{\mathrm{m}, \mathrm{n}}$ & ++ \\
\hline & $4 \mathrm{~A}$ & $92,91 \pm 0,18^{\mathrm{a}}$ & $0,22 \pm 0,02^{\mathrm{m}, \mathrm{n}, \mathrm{o}}$ & $1,61 \pm 0,07^{\mathrm{e}, \mathrm{f}, \mathrm{g}, \mathrm{h}, \mathrm{i}, \mathrm{j}}$ & $5,48 \pm 0,25^{\mathrm{i}}$ & $221 \pm 11^{1, \mathrm{~m}}$ & ++ \\
\hline & $5 \mathrm{~A}$ & $91,85 \pm 0,57^{\mathrm{a}, \mathrm{b}, \mathrm{c}, \mathrm{d}}$ & $0,32 \pm 0,03^{\mathrm{k}, \mathrm{l}, \mathrm{m}}$ & $1,94 \pm 0,06^{\mathrm{c}, \mathrm{d}, \mathrm{f}, \mathrm{g}}$ & $5,78 \pm 0,09^{\mathrm{f,g,h,i}}$ & $275 \pm 26^{\mathrm{h}, \mathrm{i}, \mathrm{j}, \mathrm{k}}$ & ++ \\
\hline & $6 \mathrm{~A}$ & $92,27 \pm 0,21^{\mathrm{a}, \mathrm{b}, \mathrm{c}, \mathrm{d}, \mathrm{e}}$ & $0,49 \pm 0,09^{\mathrm{f}, \mathrm{g}, \mathrm{h}, \mathrm{i}, \mathrm{j}}$ & $1,60 \pm 0,08^{\mathrm{f}, \mathrm{g}, \mathrm{h}, \mathrm{i}, \mathrm{j}}$ & $5,85 \pm 0,18^{\mathrm{c}, \mathrm{d}, \mathrm{e}, \mathrm{f}, \mathrm{g}, \mathrm{h}, \mathrm{i}}$ & $271 \pm 30^{\mathrm{h}, \mathrm{i}, \mathrm{j}, \mathrm{k}}$ & ++ \\
\hline \multirow{5}{*}{$5 \mathrm{~S}_{\mathrm{o}}$} & $2 \mathrm{~A}$ & $90,89 \pm 0,37^{\mathrm{a}, \mathrm{b}, \mathrm{c}, \mathrm{d}, \mathrm{e}, \mathrm{f}, \mathrm{g}, \mathrm{h}, \mathrm{i}}$ & $1,10 \pm 0,09^{\mathrm{a}}$ & $-1,77 \pm 0,09^{\mathrm{t}}$ & $5,69 \pm 0,18^{\mathrm{g}, \mathrm{h}, \mathrm{i}}$ & $200 \pm 18^{\mathrm{m}, \mathrm{o}}$ & + \\
\hline & $3 \mathrm{~A}$ & $90,78 \pm 0,59^{\text {a,b,c,d,e,e,f,g,h,i,j }}$ & $0,95 \pm 0,09^{\mathrm{b}}$ & $-1,26 \pm 0,08^{\mathrm{r}, \mathrm{s}}$ & $5,78 \pm 0,13^{\mathrm{f,g}, \mathrm{h}, \mathrm{i}}$ & $243 \pm 13^{\mathrm{j}, \mathrm{k}, \mathrm{l,n}}$ & ++ \\
\hline & $4 \mathrm{~A}$ & $91,64 \pm 0,43^{\mathrm{a}, \mathrm{b}, \mathrm{c}, \mathrm{d}, \mathrm{e}, \mathrm{f}, \mathrm{g}}$ & $1,04 \pm 0,07^{\mathrm{a}, \mathrm{b}}$ & $-1,34 \pm 0,11^{\mathrm{s}}$ & $5,97 \pm 0,09^{\mathrm{c}, \mathrm{d}, \mathrm{e}, \mathrm{f}, \mathrm{g}, \mathrm{h}}$ & $244 \pm 18^{\mathrm{j}, \mathrm{k}, \mathrm{l,n}}$ & ++ \\
\hline & $5 \mathrm{~A}$ & $91,23 \pm 0,39^{\mathrm{a}, \mathrm{b}, \mathrm{c}, \mathrm{d}, \mathrm{e}, \mathrm{f}, \mathrm{g}, \mathrm{h}}$ & $0,70 \pm 0,03^{\mathrm{c}}$ & $-0,17 \pm 0,02^{\mathrm{n}, \mathrm{o}}$ & $5,76 \pm 0,05^{\mathrm{g}, \mathrm{h}, \mathrm{i}}$ & $328 \pm 37^{\mathrm{d}, \mathrm{e}, \mathrm{f}}$ & ++ \\
\hline & $6 \mathrm{~A}$ & $92,01 \pm 0,60^{\mathrm{a}, \mathrm{b}, \mathrm{c}}$ & $0,72 \pm 0,05^{\mathrm{c}}$ & $-0,09 \pm 0,03^{\mathrm{n}, \mathrm{o}}$ & $5,85 \pm 0,17^{\mathrm{d}, \mathrm{e}, \mathrm{f}, \mathrm{g}, \mathrm{h}, \mathrm{i}}$ & $332 \pm 36^{\mathrm{d}, \mathrm{e}, \mathrm{f}}$ & + \\
\hline \multirow{5}{*}{$4 \mathrm{G}$} & $2 \mathrm{~A}$ & $90,04 \pm 0,88^{\text {c,d,e.f,g,h,i,j,k }}$ & $0,57 \pm 0,05^{\text {d,e,f,g }}$ & $2,00 \pm 0,11^{\mathrm{c}, \mathrm{d}}$ & $5,43 \pm 0,14^{\mathrm{i}}$ & $222 \pm 22^{\mathrm{j}, \mathrm{k}, \mathrm{l}, \mathrm{m}, \mathrm{o}}$ & + \\
\hline & $3 \mathrm{~A}$ & $89,23 \pm 1,68^{\mathrm{h}, \mathrm{i}, \mathrm{j}, \mathrm{k}, \mathrm{l}}$ & $0,40 \pm 0,04^{\mathrm{j}, \mathrm{k}, \mathrm{l}}$ & $1,93 \pm 0,18^{\mathrm{c}, \mathrm{d}, \mathrm{f}, \mathrm{g}}$ & $5,90 \pm 0,08^{\mathrm{c}, \mathrm{d}, \mathrm{e}, \mathrm{f}, \mathrm{g}, \mathrm{h}, \mathrm{i}}$ & $306 \pm 10^{\mathrm{d,ef,f,g,h}}$ & + \\
\hline & $4 \mathrm{~A}$ & $90,46 \pm 0,32^{\mathrm{b}, \mathrm{c}, \mathrm{d}, \mathrm{e}, \mathrm{f}, \mathrm{g}, \mathrm{gh}, \mathrm{i}, \mathrm{j}, \mathrm{k}}$ & $0,40 \pm 0,06^{\mathrm{i}, \mathrm{j}, \mathrm{k}, \mathrm{l}}$ & $2,17 \pm 0,12^{\mathrm{b}, \mathrm{c}}$ & $5,79 \pm 0,11^{\mathrm{f}, \mathrm{g}, \mathrm{h}, \mathrm{i}}$ & $328 \pm 29^{\mathrm{c}, \mathrm{d}, \mathrm{f}, \mathrm{f}, \mathrm{g}}$ & ++ \\
\hline & $5 \mathrm{~A}$ & $89,48 \pm 0,88^{\mathrm{e}, \mathrm{f}, \mathrm{g}, \mathrm{h}, \mathrm{i}, \mathrm{j}, \mathrm{k}}$ & $0,42 \pm 0,02^{\mathrm{i}, \mathrm{j}, \mathrm{k}}$ & $2,51 \pm 0,26^{\mathrm{a}, \mathrm{b}}$ & $5,75 \pm 0,20^{\mathrm{g}, \mathrm{h}, \mathrm{i}}$ & $366 \pm 31^{\mathrm{a}, \mathrm{b}, \mathrm{c}, \mathrm{d}, \mathrm{e}}$ & ++ \\
\hline & $6 \mathrm{~A}$ & $89,59 \pm 0,65^{\mathrm{d}, \mathrm{e}, \mathrm{f}, \mathrm{g}, \mathrm{h}, \mathrm{i}, \mathrm{j}, \mathrm{k}}$ & $0,33 \pm 0,02^{\mathrm{k}, \mathrm{l}, \mathrm{m}}$ & $2,60 \pm 0,20^{\mathrm{a}}$ & $5,83 \pm 0,13^{\mathrm{e}, \mathrm{f,g}, \mathrm{h}, \mathrm{i}}$ & $359 \pm 11^{\text {a,b,c,d,e,f }}$ & ++ \\
\hline \multirow{5}{*}{$5 \mathrm{G}$} & $2 \mathrm{~A}$ & $90,39 \pm 0,46^{\mathrm{b}, \mathrm{c}, \mathrm{d}, \mathrm{e}, \mathrm{f}, \mathrm{g}, \mathrm{h}, \mathrm{i}, \mathrm{j}}$ & $0,42 \pm 0,04^{\mathrm{h}, \mathrm{i}, \mathrm{j}, \mathrm{k}}$ & $1,79 \pm 0,13^{\mathrm{de,ef}, \mathrm{g}, \mathrm{h}}$ & $5,54 \pm 0,21^{\mathrm{h}, \mathrm{i}}$ & $217 \pm 19^{\mathrm{j}, \mathrm{k}, \mathrm{l}, \mathrm{m}, \mathrm{o}}$ & + \\
\hline & $3 \mathrm{~A}$ & $89,42 \pm 0,83^{\mathrm{g}, \mathrm{h}, \mathrm{i}, \mathrm{j}, \mathrm{k}, \mathrm{l}}$ & $0,42 \pm 0,04^{\mathrm{h}, \mathrm{i}, \mathrm{j}, \mathrm{k}}$ & $1,79 \pm 0,14^{\mathrm{d}, \mathrm{e}, \mathrm{f}, \mathrm{g}, \mathrm{h}}$ & $5,59 \pm 0,28^{\mathrm{i}}$ & $377 \pm 33^{\mathrm{a}, \mathrm{b}, \mathrm{c}, \mathrm{d}, \mathrm{e}}$ & ++ \\
\hline & $4 \mathrm{~A}$ & $89,15 \pm 0,41^{\mathrm{h}, \mathrm{i}, \mathrm{j}, \mathrm{k}, \mathrm{l}}$ & $0,16 \pm 0,07^{\mathrm{n}, \mathrm{o}}$ & $1,97 \pm 0,14^{\mathrm{c}, \mathrm{d}, \mathrm{f}}$ & $5,57 \pm 0,08^{\mathrm{i}}$ & $311 \pm 29^{\mathrm{f,g}, \mathrm{h}}$ & + \\
\hline & $5 \mathrm{~A}$ & $88,94 \pm 0,55^{\mathrm{h}, \mathrm{i}, \mathrm{j}, \mathrm{k}, \mathrm{l}}$ & $0,27 \pm 0,02^{1, \mathrm{~m}, \mathrm{n}}$ & $2,19 \pm 0,15^{\mathrm{b}, \mathrm{c}}$ & $6,22 \pm 0,08^{\mathrm{c}, \mathrm{d}}$ & $338 \pm 32^{\mathrm{c}, \mathrm{d}, \mathrm{e}, \mathrm{f}}$ & ++ \\
\hline & $6 \mathrm{~A}$ & $88,73 \pm 0,33^{\mathrm{i}, \mathrm{j}, \mathrm{k}, \mathrm{l}}$ & $0,31 \pm 0,03^{\mathrm{k}, \mathrm{l}, \mathrm{m}}$ & $2,58 \pm 0,27^{\mathrm{a}}$ & $6,31 \pm 0,21^{\mathrm{b}, \mathrm{c}}$ & $410 \pm 45^{\mathrm{a}}$ & + \\
\hline \multirow{3}{*}{$4 S_{c}$} & $2 \mathrm{~A}$ & $91,03 \pm 1,04^{\mathrm{a}, \mathrm{b}, \mathrm{c}, \mathrm{d}, \mathrm{e}, \mathrm{f}, \mathrm{g}, \mathrm{h}}$ & $0,72 \pm 0,03^{\mathrm{c}}$ & $-1,06 \pm 0,08^{\mathrm{q}, \mathrm{r}, \mathrm{s}}$ & $5,75 \pm 0,10^{\mathrm{g}, \mathrm{h}, \mathrm{i}}$ & $236 \pm 25^{\mathrm{k}, \mathrm{l,m}}$ & + \\
\hline & $3 \mathrm{~A}$ & $90,71 \pm 0,50^{\mathrm{a}, \mathrm{b}, \mathrm{c}, \mathrm{d}, \mathrm{e}, \mathrm{f}, \mathrm{g}, \mathrm{gh,i,j}}$ & $0,73 \pm 0,04^{\mathrm{c}}$ & $-0,85 \pm 0,04^{\mathrm{p}, \mathrm{q}}$ & $5,83 \pm 0,10^{\mathrm{e}, \mathrm{f}, \mathrm{g}, \mathrm{h}, \mathrm{i}}$ & $273 \pm 22^{\mathrm{h}, \mathrm{i}, \mathrm{j}, \mathrm{k}}$ & + \\
\hline & $4 \mathrm{~A}$ & $89,44 \pm 1,04^{\mathrm{f}, \mathrm{g}, \mathrm{h}, \mathrm{i}, \mathrm{j}, \mathrm{k}}$ & $0,66 \pm 0,06^{\mathrm{cd, \textrm {d }}}$ & $-0,19 \pm 0,04^{\circ}$ & $6,02 \pm 0,14^{\mathrm{c}, \mathrm{d}, \mathrm{e}, \mathrm{f}, \mathrm{g}}$ & $281 \pm 24^{\mathrm{g}, \mathrm{h}, \mathrm{j}}$ & + \\
\hline
\end{tabular}




\section{9 a 22 de outubro de 2014}

Florianópolis/SC

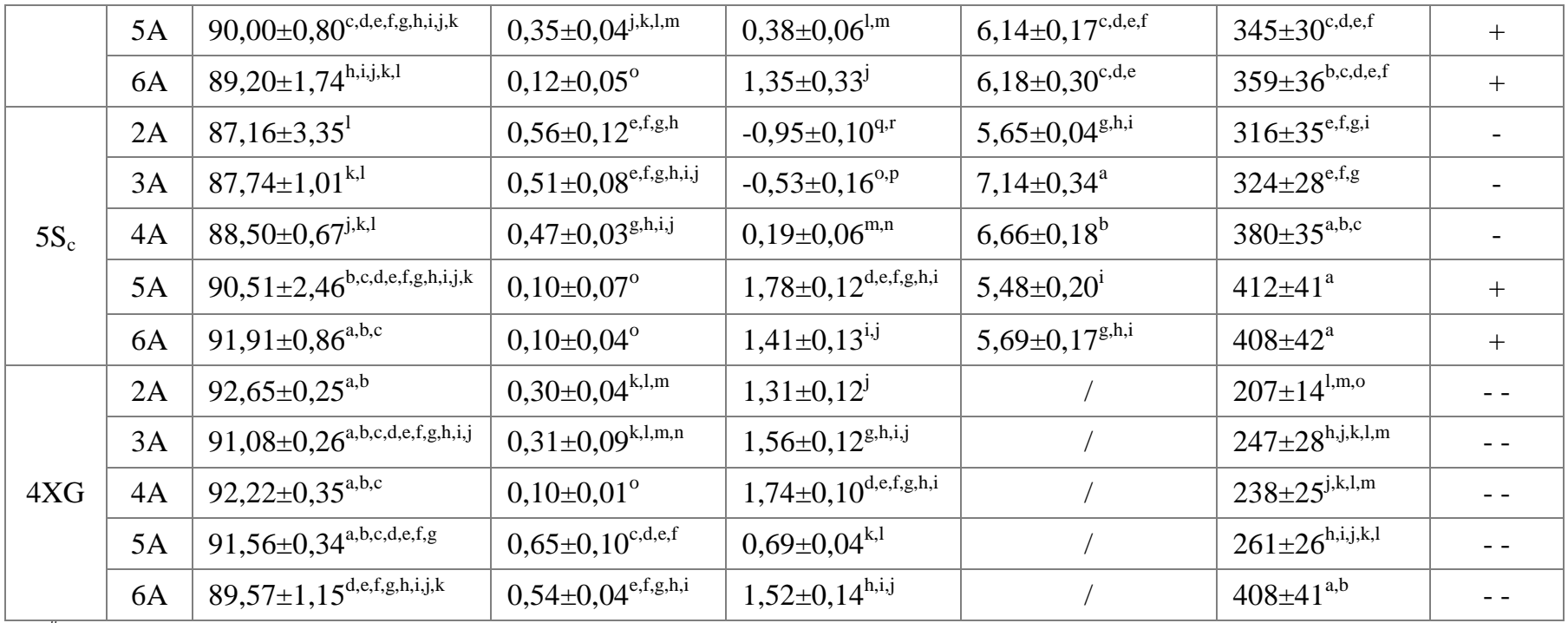

${ }^{\#}$ Os números indicam a quantidade $(\mathrm{g} / 100 \mathrm{~g})$ dos plastificantes sorbitol (So), glicerol $(\mathrm{G})$, sacarose (Sc) e xarope de glicose (XG) e amido de pinhão (A) utilizados em cada formulação. Os símbolos para aparência representam boa aparência e maleabilidade (+ +), maleável (+), não maleável (-) e má aparência e não maleável (- -). (/) Não foi possível medir.

Letras iguais na mesma coluna não apresentam diferença significativa pelo teste de Tukey $(\mathrm{p}>0,05)$.

\section{CONCLUSÃO}

A partir do estudo realizado pôde-se perceber que sacarose e xarope de glicose não têm ação plastificante quando utilizados separadamente, inviabilizando sua aplicação. Os filmes com glicerol e sorbitol apresentaram maior transparência e maleabilidade e, quanto maior o percentual de amido de pinhão, menor transparência e maior a espessura. Ainda, alguns filmes com glicerol apresentaram aspecto pegajoso e com tendência a maior absorção de umidade. Assim, com base nos plastificantes utilizados nas condições do estudo, conclui-se que o sorbitol é o mais indicado para esta aplicação.

\section{REFERÊNCIAS BIBLIOGRÁFICAS}

AL-HASSAN, A. A. e NORZIAH, M. H. Starch-gelatin edible films: Water vapor permeability and mechanical properties as affected by plasticizers. Food Hydrocolloids, v.26, n.1, p.108117. 2012.

BELLO-PÉREZ， L. A., GARCÍA-SUÁREZ， F. J., MÉNDEZ-MONTEALVO, G., NASCIMENTO, J. R. O., LAJOLO, F. M. e CORDENUNSI, B. R. Isolation and characterization of starch from seeds of Araucaria brasiliensis: a novel starch for application in food industry. Starch - Stärke, v.58, n.6, p.283-291. 2006. 
CHILlo, S., FlORES, S., MASTROMATTEO, M., CONTE, A., GERSCHENSON, L. e DEL NOBILE, M. A. Influence of glycerol and chitosan on tapioca starch-based edible film properties. J Food Eng, v.88, n.2, p.159-168. 2008.

CORDENUNSI, B. M.; MENEZES, E. W.; GENOVESE, M. I.; COLLI, C.; SOUZA, A. G. e LAJOLO, F. M. Chemical composition and glycemic index of brazilian pine (Araucaria angustifolia) seeds. J Agr Food Chem, v.52, n.11, p.3412-3416. 2004.

DAUDT, R. M., KÜLKAMP-GUERREIRO, I. C., CLADERA-OLIVERA, F., THYS, R. C. S. e MARCZAK, L. D. F. Determination of properties of pinhão starch: Analysis of its applicability as pharmaceutical excipient. Ind Crop Prod, v.52, p.420-429. 2014.

DOS SANTOS, A. J., CORSO, N. M., MARTINS, G. e BITTENCOURT, E. Aspectos produtivos e comerciais do pinhão no Estado do Paraná. Floresta, v.32, n.2, p.163-169. 2002.

FAKHOURY, F. M., MARTELli, S. M., BERTAN, L. C., YAMASHITA, F., MEI, L. H. I. e QUEIROZ, F. P. C. Edible films made from blends of manioc starch and gelatin - Influence of different types of plasticizer and different levels of macromolecules on their properties. LWT - Food Sci Technol, v.49, n.1, p.149-154. 2012.

GARCIA, M. A., PINOTTI, A. e ZARITZKY, N. E. Physicochemical, Water Vapor Barrier and Mechanical Properties of Corn Starch and Chitosan Composite Films. Starch - Stärke, v.58, n.9, p.453-463. 2006.

GIALAMAS, H., ZINOVIADOU, K. G., BILIADERIS, C. G. e KOUTSOUMANIS, K. P. Development of a novel bioactive packaging based on the incorporation of Lactobacillus sakei into sodium-caseinate films for controlling Listeria monocytogenes in foods. Food Res Int, v.43, n.10, p.2402-2408. 2010.

KAMMOUN, M., HADDAR, M., KALLEL, T. K., DAMMAK, M. e SAYARI, A. Biological properties and biodegradation studies of chitosan biofilms plasticized with PEG and glycerol. Int J Biol Macromol, v.62, n.0, p.433-438. 2013.

KECHICHIAN, V., DITCHFIELD, C., VEIGA-SANTOS, P. e TADINI, C. C. Natural antimicrobial ingredients incorporated in biodegradable films based on cassava starch. $L W T$ - Food Sci Technol, v.43, n.7, p.1088-1094. 2010.

MALI, S., GROSSMANN, M. V. R. E., GARCÃ-A, M. A., MARTINO, M. N. e ZARITZKY, N. E. Effects of controlled storage on thermal, mechanical and barrier properties of plasticized films from different starch sources. Journal of Food Engineering, v.75, n.4, p.453-460. 2006. 
MANiglia, B. C., DOMingos, J. R., DE PAUlA, R. L. e TAPIA-BLÁCidO, D. R. Development of bioactive edible film from turmeric dye solvent extraction residue. $L W T$ Food Sci Technol, v.56, n.2, p.269-277. 2014.

MINOLTA. Comunicação precisa da cor: controle de qualidade da percepção à instrumentação. Japão: Minolta Corporation. 59 p. 1998.

SALMIERI, S. e LACROIX, M. Physicochemical Properties of Alginate/Polycaprolactone-Based Films Containing Essential Oils. J Agr Food Chem, v.54, n.26, p.10205-10214. 2006.

SOBRAL, P. J. A., MENEGAlli, F. C., HUBINGER, M. D. e ROQUES, M. A. Mechanical, water vapor barrier and thermal properties of gelatin based edible films. Food Hydrocolloids, v.15, n.4-6, p.423-432. 2001.

SORRENTINO, A., GORRASI, G. e VITTORIA, V. Potential perspectives of bionanocomposites for food packaging applications. Trends Food Sci Tech, v.18, n.2, p.84-95. 2007.

SOUZA, A. C., BENZE, R., FERRÃO, E. S., DITCHFIELD, C., COELHO, A. C. V. e TADINI, C. C. Cassava starch biodegradable films: Influence of glycerol and clay nanoparticles content on tensile and barrier properties and glass transition temperature. LWT - Food Sci Technol, v.46, n.1, p.110-117. 2012.

SPADA, J. C., MARCZAK, L. D. F., TESSARO, I. C. e NOREÑA, C. P. Z. Microencapsulation of $\beta$-carotene using native pinhão starch, modified pinhão starch and gelatin by freezedrying. Int J Food Sci Tech, v.47, n.1, p.186-194. 2012.

THYS, R. C. S., NOREÑA, C. P. Z., MARCZAK, L. D. F., AIRES, A. G. e CLADERAOLIVERA, F. Adsorption isotherms of pinhão (Araucaria angustifolia seeds) starch and thermodynamic analysis. J Food Eng, v.100, n.3, p.468-473. 2010.

THYS, R. C. S., WESTFAHL, H., NOREÑA, C. P. Z., MARCZAK, L. D. F., SILVEIRA, N. D. P. e CARDOSO, M. B. Effect of the Alkaline Treatment on the Ultrastructure of C-Type Starch Granules. Biomacromolecules, v.9, n.7, p.1894-1901. 2008.

VÁSCONEZ, M. B., FLORES, S. K., CAMPOS, C. A., ALVARADO, J. e GERSCHENSON, L. N. Antimicrobial activity and physical properties of chitosan-tapioca starch based edible films and coatings. Food Res Int, v.42, n.7, p.762-769. 2009. 